\title{
Towards the Architecture of the TOC Protein Complex
}

\author{
Karthik Srinivasan ${ }^{\mathrm{a}, \mathrm{b}}$, Runrun Wua ${ }^{\mathrm{a}}$, Adrian Gonzalez ${ }^{\mathrm{a}}$, Nicholas Noinaja,c \\ aDepartment of Biological Sciences, Purdue University, 240 S. Martin Jischke Dr. West \\ Lafayette, IN, 47907 \\ bsriniv67@purdue.edu \\ cnnoinaj@purdue.edu
}

Protein translocation into plastids is essential for the pathogenesis of human parasites like malaria and for essential processes such as photosynthesis in chloroplasts. Chloroplasts are one of the best studied plastid systems and hence are a good model to study these complex translocation machineries and their mechanisms. Through endosymbiotic evolution, chloroplast proteins became nuclearly encoded over time, which necessitated the genesis of a translocon system that could transport cargo proteins across the organelle membranes. The TOC and TIC protein complexes present at the outer and inner chloroplast membrane respectively, together orchestrate this vital translocation system. Our lab seeks to elucidate the structural architecture of the TOC complex in order to gain mechanistic insights into protein translocation in plastids. Toc75 is a $\beta$-barrel protein that forms the channel of the TOC translocon and our lab has previously reported the first structure of Toc75 consisting of the soluble domain but lacking the membrane-embedded domain. While this work provided insight into the chaperone-like activity of the soluble domain, it gave no clues about the translocation role of Toc75. Recently though, we have to purified, expressed, and crystallised a full length construct of Toc75. Current efforts are directed towards crystal optimisation. Future directions include generation of transgenic plants expressing affinity tagged versions of the TOC complex components and production of synthetic antibodies, both for the purpose of purifying the TOC complex directly from plants for structural characterisation. 